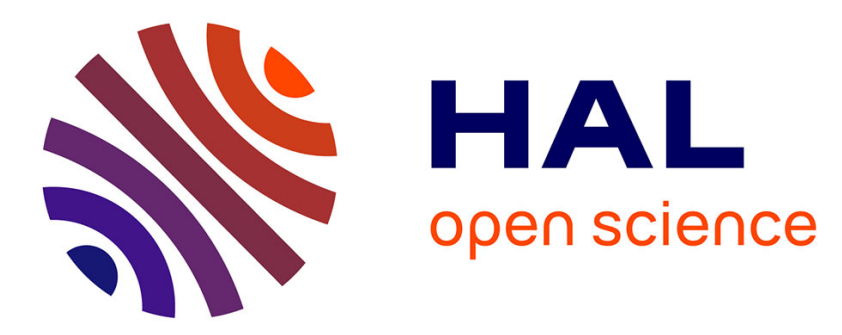

\title{
Magnetic-field assisted performance of InGaAs/GaAsSb terahertz quantum cascade lasers
}

Simon Maëro, Louis-Anne de Vaulchier, Yves Guldner, Christoph Deutsch, Michael Krall, Tobias Zederbauer, Gottfreid Strasser, Karl Unterrainer

\section{- To cite this version:}

Simon Maëro, Louis-Anne de Vaulchier, Yves Guldner, Christoph Deutsch, Michael Krall, et al.. Magnetic-field assisted performance of InGaAs/GaAsSb terahertz quantum cascade lasers. Applied Physics Letters, 2013, pp.051116. hal-00911496

\section{HAL Id: hal-00911496 \\ https://hal.science/hal-00911496}

Submitted on 29 Nov 2013

HAL is a multi-disciplinary open access archive for the deposit and dissemination of scientific research documents, whether they are published or not. The documents may come from teaching and research institutions in France or abroad, or from public or private research centers.
L'archive ouverte pluridisciplinaire HAL, est destinée au dépôt et à la diffusion de documents scientifiques de niveau recherche, publiés ou non, émanant des établissements d'enseignement et de recherche français ou étrangers, des laboratoires publics ou privés. 


\title{
Magnetic-field assisted performance of InGaAs/GaAsSb terahertz quantum cascade lasers
}

\author{
Simon Maëro, ${ }^{1}$ Louis-Anne de Vaulchier, ${ }^{1,} *$ Yves Guldner, ${ }^{1}$ Christoph Deutsch, ${ }^{2}$ \\ Michael Krall, ${ }^{2}$ Tobias Zederbauer, ${ }^{3}$ Gottfried Strasser, ${ }^{3}$ and Karl Unterrainer ${ }^{2}$ \\ ${ }^{1}$ Laboratoire Pierre Aigrain, Ecole Normale Supérieure, \\ CNRS (UMR 8551), Université Pierre et Marie Curie, \\ Université Paris Diderot, 24 rue Lhomond, 75231 Paris Cedex 05, France \\ ${ }^{2}$ Photonics institute and Center for Micro- and Nanostructures, \\ Vienna University of Technologies, Gusshausstrasse 27-29, 1040 Vienna, Austria \\ ${ }^{3}$ Institude for Solid State Electronics and Center for Micro- and Nanostructures, \\ Vienna University of Technologies, Floragasse 7, 1040 Vienna, Austria
}

(Dated: July 15, 2013 - v 1.0)

\begin{abstract}
We report on a magnetic-field investigation of a $\operatorname{In}_{0.53} \mathrm{Ga}_{0.47} \mathrm{As} / \mathrm{GaAs}_{0.51} \mathrm{Sb}_{0.49}$ terahertz quantum cascade laser. Owing to the suppression of inter-Landau level non-radiative scattering, the device performances are strongly improved at high magnetic field. Working temperature up to $190 \mathrm{~K}$ and current threshold of $450 \mathrm{~A} / \mathrm{cm}^{2}$ are measured at 11 teslas, comparable to the state-of-the-art GaAs/AlGaAs terahertz lasers. The nominally symmetric three-well structure presents significantly better performance with negative bias polarisation because of the inverted-interface roughness impact on the laser action.
\end{abstract}

Quantum cascade lasers (QCL) are promising sources in the terahertz $(\mathrm{THz})$ range but their use in applications is limited by their temperature performance because it is difficult to maintain a population inversion between closely spaced electronic levels $(1 \mathrm{THz} \sim 4 \mathrm{meV})$. Among the various proposed QCL designs, the three-well resonant phonon based structure usually shows the best temperature behaviour ${ }^{1,2}$.The dipole matrix element of the radiative transition and the upper state lifetime are the key-parameters to obtain high temperature operation. By improving the QCL design, operating temperatures up to $200 \mathrm{~K}$ have been reported in GaAs/AlGaAs structures ${ }^{2}$. Nevertheless, room temperature operation seems very difficult to reach by further optimization in the GaAs/AlGaAs system. Recently, QCLs based on InGaAs/GaAsSb type II heterostructures have been proposed ${ }^{3}$ in order to improve the characteristics of the device, in particular because of the low electron effective mass in this system and its suitable conduction band offset. However, it has been shown ${ }^{4}$ that the strong effects of asymmetric interfaces in these heterostructures, with different group $\mathrm{V}$ elements for wells and barriers (As and $\mathrm{Sb}$ ), is a limitation which must be taken into account.

It is well-known that the application of a magnetic field parallel to the growth axis is a very valuable technique to characterize the QCL and to evaluate the involved scattering mechanisms ${ }^{5-7}$. In this paper, we have investigated a three-well $\mathrm{In}_{0.53} \mathrm{Ga}_{0.47} \mathrm{As} / \mathrm{GaAs}_{0.51} \mathrm{Sb}_{0.49}$ QCL under magnetic field up to $15 \mathrm{~T}$ in the temperature range $4-220 \mathrm{~K}$. The QCL is the same structure as Sample 2 of Ref. 4 with a symmetric active region, so that the bias polarity could be applied either positive or negative. Starting from the first quantum well, the layer sequence in $\AA$ is as follows 30/133/10/133/30/20/188/20 (the barrier widths are represented in bold types). The Si doping density is $1 \times 10^{16} \mathrm{~cm}^{-3}$ in the underlined section. The structure has been grown by MBE as described in Ref 4 and consists of 170 three-well periods. The active region was then processed in a double metal waveguide and the ridges $(50 \mu \mathrm{m} \times 1 \mathrm{~mm})$ were formed by lithography and dry etching. The conduction band diagram calculated for an applied electric field of $9.8 \mathrm{kV} / \mathrm{cm}(55 \mathrm{meV}$ (a)
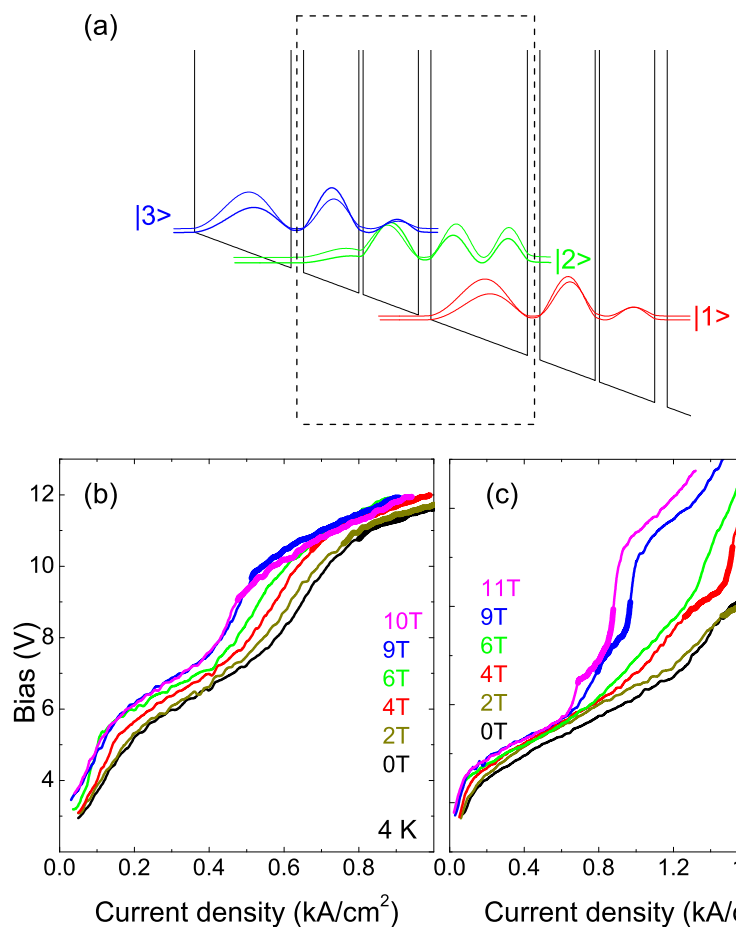

(c)

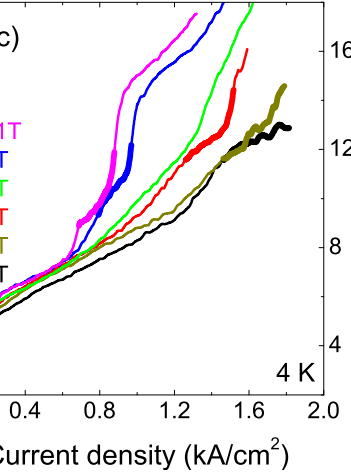

FIG. 1: (a) Conduction band diagram of the three-well structure using a one-dimensional Schrödinger solver at a bias of $9.8 \mathrm{kV} / \mathrm{cm}$. The radiative transition occurs between $|3\rangle$ and $|2\rangle$. The dashed rectangle indicates the three-well period. (b) Voltage versus current density $J$ for a $50 \mu \mathrm{m} \times 1 \mathrm{~mm}$ ridge. Characteristics are measured for various $B$ at $4 \mathrm{~K}$ for negative bias. The bold line sections indicate alignment regions where the laser operates. (c) Same as (b) for positive bias.

per period) is presented on Fig. 1(a). A one-band model is used, including the conduction band non-parabolicity effect in InGaAs and GaAsSb. Band-edge electron effective masses of $0.043 m_{0}$ and $0.045 m_{0}$ are taken for $\operatorname{In}_{0.53} \mathrm{Ga}_{0.47} \mathrm{As}$ and $\mathrm{GaAs}_{0.51} \mathrm{Sb}_{0.49}$ respectively as well as a conduction band offset of $360 \mathrm{meV}^{8}$. The laser optical transition occurs be- 


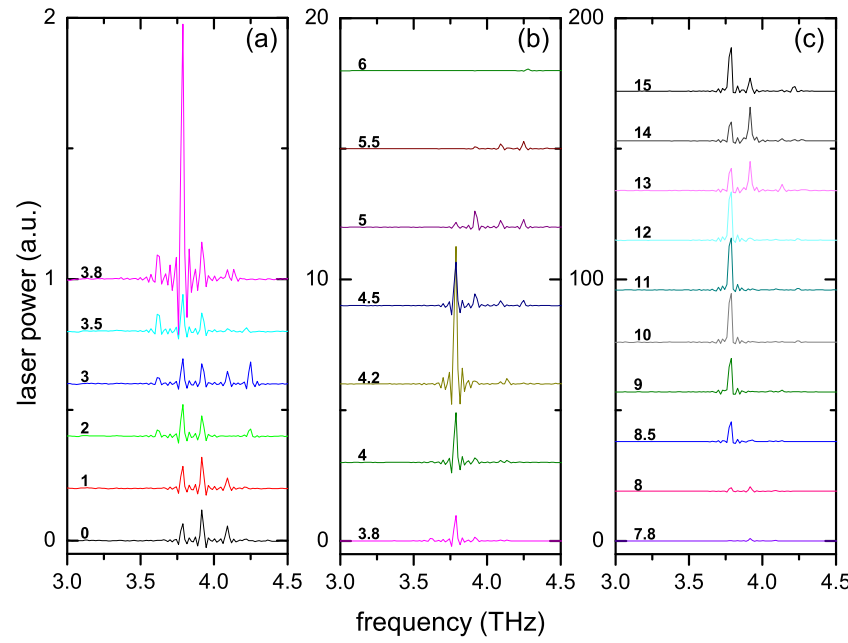

FIG. 2: Evolution of the QCL emission lines as a function of $B$ (in teslas) for negative bias at $4 \mathrm{~K}$. The vertical axis between each panel (a), (b), (c) differs by a factor of ten.

tween subbands $|3\rangle$ and $|2\rangle$ and is calculated to be $15.6 \mathrm{meV}$ $(3.8 \mathrm{THz})$. Electrons in the lower lasing level $|2\rangle$ relax to level $|1\rangle$ through electron-LO phonon scattering (the LO phonon energy in InGaAs being $\hbar \omega_{L O}=34 \mathrm{meV}$ ), which guarantees an efficient depopulation of $|2\rangle$. As shown in Ref. 4 , the nominally symmetric structure is in fact asymmetric because of the asymmetry of the normal and inverted InGaAs/GaAsSb interfaces (the normal interface corresponds to the switching during growth from the well to barrier and vice versa for the inverted one). TEM analysis shows that the roughness of the inverted interface is much larger than that of the normal one as it is usually observed in this material system with different group $\mathrm{V}$ elements for wells and barriers. Depending on the bias polarity of the structure (positive or negative), the electron wave function shown on Fig. 1(a) are either pushed toward the rougher or smoother interfaces, leading to different scattering rates for the two polarities. In the experimental setup, a positive bias leads to an electron flow towards the rougher inverted interface and a negative bias to an electron flow towards the smoother normal interface. The application of a strong perpendicular magnetic field $B$ changes the twodimensional parabolic dispersion of the relevant energy levels $|i\rangle$ into a set of discrete quasi-equidistant Landau levels (LLs) $|i, n\rangle$, where $n=0,1$, is the LL index. LL energies are calculated using the same one-band model including the non-parabolicity effect as for $B=0$ and the calculated fan chart of the LLs $|2, n\rangle$ and $|3,0\rangle$ is shown on Fig. 3(a). The width of each Landau level depends on the electron scattering rate and is therefore expected to be different for positive or negative bias polarity.

The voltage versus current density characteristics at various magnetic fields are presented on Fig. 1(b) and 1(c) for negative and positive polarity respectively. For negative bias, as $B$ is increased from 0 to $15 \mathrm{~T}$, the onset of the zone where the laser action takes place (alignment zone) moves continuously from $J=0.8$ to $0.45 \mathrm{kA} / \mathrm{cm}^{2}$. Note that the structure alignment is

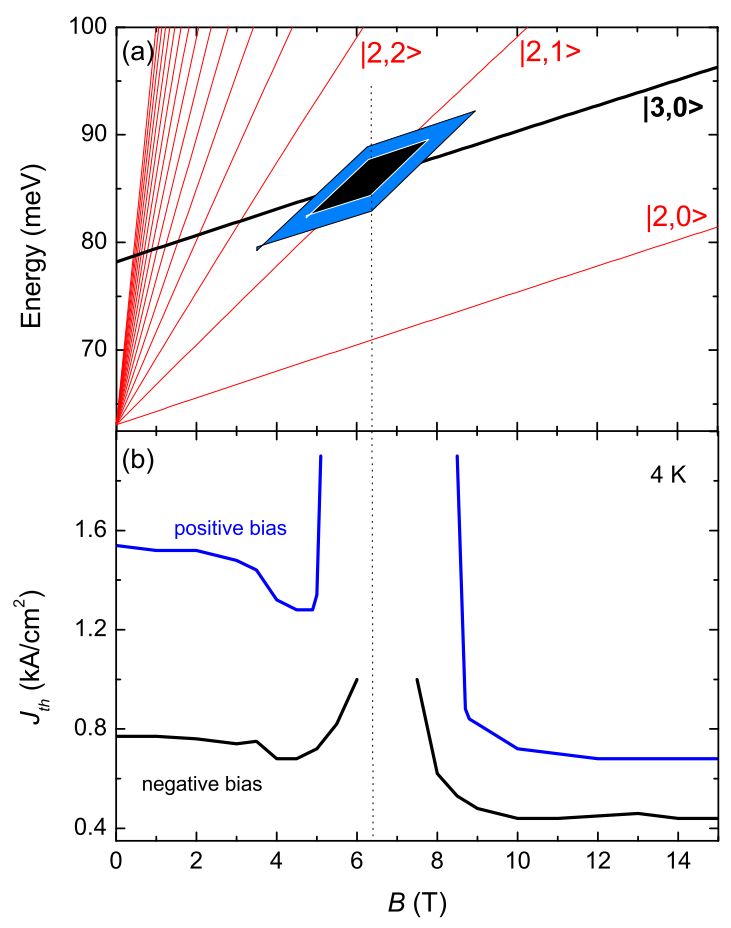

FIG. 3: (a) Landau level fan chart of the active region. The lower LL $|2,1\rangle$ crosses the upper lasing level $|3,0\rangle$ around $6.4 \mathrm{~T}$. The LL crossing domain, taking into account the finite width of the levels, is schematically represented by the black and blue diamonds for negative and positive bias respectively (see text). (b) Threshold current density versus $B$ measured at $4 \mathrm{~K}$ for negative (black) and positive (blue) bias.

preserved at $J=0.8 \mathrm{kA} / \mathrm{cm}^{2}$ for the whole $B$ range. Results for positive bias are quite different, as the characteristics shift versus $B$ is more important. The onset of the alignment zone drops from 1.6 to $0.7 \mathrm{kA} / \mathrm{cm}^{2}$ and the width of the alignment zone dramatically shrinks as compared to the negative bias. For example at $B=11 \mathrm{~T}$, the alignment zone only occurs in the vicinity of $J=0.8 \mathrm{kA} / \mathrm{cm}^{2}$. It is therefore no longer possible to define a fixed current where the alignment is preserved in the whole $B$ range.

The QCL laser emission was measured using a fast Fourier transform spectrometer with a resolution of $1 \mathrm{~cm}^{-1}(1 \mathrm{THz}=$ $33 \mathrm{~cm}^{-1}$ ) and a Si far-infrared bolometer. The emission at $4 \mathrm{~K}$ is shown on Fig. 2 (a), (b), (c) for various magnetic fields, negative bias and a fixed current density $J=0.8 \mathrm{kA} / \mathrm{cm}^{2}$. The device was operated in pulse mode with $2 \mu$ s long pulses at a repetition rate of $10 \mathrm{kHz}$. Note that the vertical axis between each panel (a), (b), (c) differs by a factor ten, i.e. a factor of 100 between (a) and (c). Essentially two points are noticeable: (i) a strong enhancement of the laser line intensity as $B$ is increased; (ii) a switch-off of the laser action between 6 and $7.5 \mathrm{~T}$. The main laser emission is observed at $3.8 \mathrm{THz}$ $(15.6 \mathrm{meV})$ in good agreement with calculated energy levels shown on Fig. 1. The $B$-enhancement of the line is consistent with the $V(J)$ characteristic behaviour shown on Fig. 1(b), as the current density $J$ is close to the onset of the alignment zone at $B=0$ and in the middle of this zone at high $B$. A 


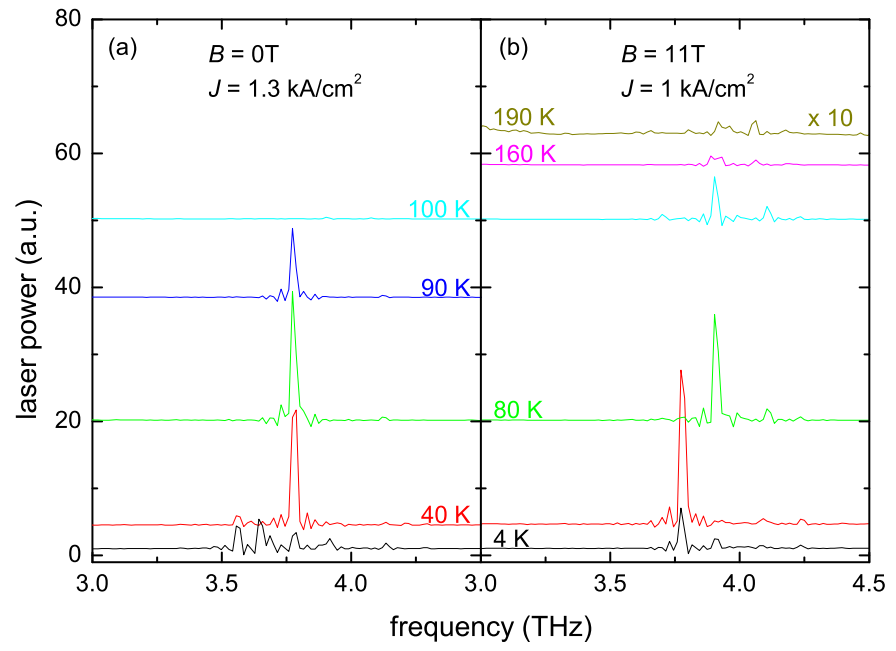

FIG. 4: (a) Laser emission for various temperatures at $B=0$ for negative bias and $J=1.3 \mathrm{kA} / \mathrm{cm}^{2}$. The device operates up to $\sim$ $100 \mathrm{~K}$. (b) Laser emission at various temperatures at $B=11 \mathrm{~T}$ for negative bias and $J=1 \mathrm{kA} / \mathrm{cm}^{2}$. The device operates up to $\sim$ $190 \mathrm{~K}$. For clarity, the curve at $190 \mathrm{~K}$ is magnified by a factor 10 .

factor of 100 is measured between 0 and $15 \mathrm{~T}$ for the main line. Additional strong laser lines appear between 3.6 and $4.2 \mathrm{THz}$. The extinction between 6 and $7.5 \mathrm{~T}$ can be explained by the crossing of $\mathrm{LL}|2,1\rangle$ and $|3,0\rangle$ occurring around $6.4 \mathrm{~T}$ (see Fig. 3(a)). It is well-known that the lifetime of the upper lasing level is significantly reduced in the vicinity of the LL crossing because the non-radiative scattering is increased ${ }^{5,6}$. The $B$-domain of the extinction characterizes the width of the LLs. The black diamond on Fig. 3(a) represents schematically the crossing area taking into account a LL width of $4 \mathrm{meV}$, in good agreement with calculations of broadening effects due to alloy scattering in $\mathrm{In}_{0.53} \mathrm{Ga}_{0.47}$ As $\mathrm{QCL}^{9}$. The thresholdcurrent density $J_{t h}$ of the laser action, measured at $4 \mathrm{~K}$, is shown on Fig. 3(b). $J_{t h}$ is around $750 \mathrm{~A} / \mathrm{cm}^{2}$ for $B<5 \mathrm{~T}$ and drops down to $450 \mathrm{~A} / \mathrm{cm}^{2}$ for high $B$. The significant improvement of the laser action at high magnetic field is usually observed in the THz QCLs ${ }^{11,12}$. In fact, for $B>8 \mathrm{~T}$, scattering from the upper lasing level $|3,0\rangle$ to the $|2, n\rangle$ LLs is suppressed for any value of the LL index $n$. The upper lasing level lifetime increases, and therefore the gain coefficient too. The black diamond on Fig. 3(a) fairly corresponds to the $B$ domain where the laser switches off. Similar measurements were done for a positive bias. The current threshold $J_{t h}$ is shown on Fig. 3(b) (blue line). $J_{t h}$ is typically twice larger than measured for negative bias in the whole $B$-range. The energy position of the laser lines is similar, around $3.8 \mathrm{THz}$. The most interesting point is that the laser action quenches now between 5 and $8.5 \mathrm{~T}$ due to the crossing of LL $|2,1\rangle$ and $|3,0\rangle$. The larger $B$-domain of the laser extinction (3.5 T instead of $1.5 \mathrm{~T}$ for negative bias) can be explained by the larger LL width in that case where the electron wave functions are pushed towards the rougher inverted interfaces. The current threshold $J_{t h}$ is proportional to the total scattering rate $1 / \tau$ of the upper lasing level ${ }^{10}$. Results shown on Fig. 3(b) indicate that $1 / \tau$ is roughly two time larger for positive bias than for a negative one. If one considers, following Ando et. al. ${ }^{13}$, a dependance of the LL-width $\Gamma \propto \sqrt{B / \tau}$, one deduces that $\Gamma$ is larger by a factor of $\sqrt{2}$ for positive bias. The blue diamond on Fig. 3(a) represents schematically the crossing area taking into account a LL width of $4 \sqrt{2} \mathrm{meV}$. Again, the diamond position fairly corresponds to the $B$-domain where the laser switches off. The significantly better laser performance with negative bias polarity is a clear indication that the interface roughness scattering has a major impact on the laser action.

We have studied the laser emission as a function of temperature in the range $T=4-220 \mathrm{~K}$ under magnetic field. It is known that the laser action at high temperature can be improved under a strong magnetic field. For instance, laser action at $3 \mathrm{THz}$ up to $225 \mathrm{~K}$ was reported at $B=19.3 \mathrm{~T}$ in a $\mathrm{GaAs} / \mathrm{Al}_{0.15} \mathrm{Ga}_{0.85} \mathrm{As} \mathrm{QCL}^{12}$. Here we present only the results for negative bias polarity where better performances are expected. Typical results at $B=0$ and $11 \mathrm{~T}$ are shown on Fig. 4 for current density $J=1.3 \mathrm{kA} / \mathrm{cm}^{2}$ and $1 \mathrm{kA} / \mathrm{cm}^{2}$ respectively. Again, the device was operated in pulse mode with $2 \mu$ s long pulses at a repetition rate of $10 \mathrm{kHz}$. The laser line at $B=0$ vanishes for $T>100 \mathrm{~K}$ while a weak laser action is still measured for $B=11 \mathrm{~T}$ at $T=190 \mathrm{~K}$ and vanishes completely only at $T=195 \mathrm{~K}$. The use of OD confinement in QCL has been proposed as an efficient mechanism to suppress non-radiative relaxation and achieve higher temperature operation $^{12}$. A magnetic field, changing the $2 \mathrm{D}$ subband into a set of discrete 0D-like LLs, is therefore an effective tool to improve the QCL performances ${ }^{6,11}$. One might note on Fig. 4 that the laser action at $4 \mathrm{~K}$ is smaller than that measured at $40 \mathrm{~K}$ and $80 \mathrm{~K}$. Indeed, we present the emission at a fixed current density in the whole investigated temperature range. This current density is chosen to optimize the laser emission at high temperature, thus the device is not operated at maximum output power at $4 \mathrm{~K}$. Finally, we have performed similar temperature measurements for positive bias, which show deteriorated performances with a working operation limited at $50 \mathrm{~K}$ for $B=0$ and $135 \mathrm{~K}$ for $B=11 \mathrm{~T}$.

In conclusion, we have investigated under magnetic field a $\mathrm{In}_{0.53} \mathrm{Ga}_{0.47} \mathrm{As} / \mathrm{GaAs}_{0.51} \mathrm{Sb}_{0.49}$ symmetric QCL emitting at $3.8 \mathrm{THz}$ for positive and negative bias. Reasonable device performances (laser current threshold and working temperature) are obtained for a negative bias polarity where electrons are moving against the smoother normal interface. Owing to the suppression of inter Landau level non-radiative scattering, we demonstrate unambiguously that the device performances are improved at high magnetic field. For instance, working temperature up to $190 \mathrm{~K}$ and current threshold as low as $450 \mathrm{~A} / \mathrm{cm}^{2}$ were measured at $11 \mathrm{~T}$. The significantly better laser performance with negative bias polarity, in particular the demonstration of a smaller LL width, is a clear indication that the interface roughness scattering has a major impact on the laser action. Finally, this work suggests that such QCL with a better growth quality could be good candidates to challenge the state-of-the-art GaAs/AlGaAs THz QCLs for higher temperature laser operation.

The Laboratoire Pierre Aigrain is a "Unité Mixte de Recherche" (UMR 8551) between École Normale Supérieure, 
CNRS, the University Pierre et Marie Curie (Paris 6) and the University Paris-Diderot (Paris 7). The authors would like to thank G. Bastard for very valuable and fruitful discussions and acknowledge the support by the Austrian Science Fund FWF (SFB IR-ON F25 and DK CoQuS W1210).
* Electronic address: louis-anne.devaulchier@lpa. ens.fr

${ }^{1}$ H. Luo, S. R.Laframboise, Z. R. Wasilewski, G. C. Aers, H. C. Liu, Appl. Phys. Lett. 90, 041112 (2007)

${ }^{2}$ S. Fathololoumi, E. Dupont, C. W. I. Chan, Z. R. Wasilewski, S. R. Laframboise, D. Ban, A. Matyas, C. Jirauschek, Q. Hu, H. C. Liu, Opt. Express 20, 3866 (2012)

${ }^{3}$ C. Deutsch, A. Benz, H. Detz, P. Klang, M. Nobile, A. M. Andrews, W. Schrenk, T. Kubis, P. Vogl, G. Strasser, K. Unterrainer, Appl. Phys. Lett. 97, 261110 (2010)

${ }^{4}$ C. Deutsch, H. Detz, T. Zederbauer, A. M. Andrews, P. Klang, T. Kubis, G. Klimeck, M. E. Schuster, W. Schrenk, G. Strasser, K. Unterrainer, Opt. Express 21, 7209-7215 (2013)

5 A. Leuliet, A. Vasanelli, A. Wade, G. Fedorov, D. Smirnov, G. Bastard, and C. Sirtori, Phys. Rev. B 73, 085311 (2006)

${ }^{6}$ N. Péré-Laperne, L.-A. de Vaulchier, Y. Guldner, G. Bastard, G. Scalari, M. Giovannini, J. Faist, A. Vasanelli, S. Dhillon, and C.
Sirtori, Appl. Phys. Lett. 91, 062102 (2007)

7 V. Tamosiunas, R. Zobl, J. Ulrich, K. Unterrainer, R. Colombelli, C. Gmachl, K. West, L. Pfeiffer, and F. Capasso, Appl. Phys. Lett. 83, 3873 (2003)

${ }^{8}$ M. Nobile, H. Detz, E. Mujagié, A. M. Andrews, P. Klang, W. Schrenk, G. Strasser, Appl. Phys. Lett. 95, 041102 (2009)

9 N. Regnault, R. Ferreira, and G. Bastard, Phys. Rev. B 76, 165121 (2007)

10 J. Alton, S. Barbieri, J. Fowler, H. Beere, J. Miscat, E. Linfield, D. Ritchie, G. Davies, R. Köhler, and A. Tredicucci, Phys. Rev. B 68, 081203 (2003).

11 G. Scalari, S. Blaser, J. Faist, H. Beere, E. Linfield, D. Ritchie, Phys. Rev. Lett. 93, 237403 (2004)

12 A. Wade, G. Fedorov, D. Smirnov, S. Kumar, B. S. Williams, Q. $\mathrm{Hu}$, J. L.Reno, nature photon. 3, 41-46 (2008)

13 T. Ando, Y. Uemura, J. Phys. Soc. Japan 36, 959 (1974) 